\title{
UNA LUCERNA ROMANA DE BRONCE, CON DECORACION ZOOMORFA, PROCEDENTE DE FUENLABRADA DE LOS MONTES (BADAJOZ)
}

\author{
Desiderio VAQUERIZO GIL * \\ $M^{a}$. Teresa AMARE TAFALLA *
}

\section{Resumen}

Damos a conocer en esta breve nota una lucerna romana de bronce, con decoración zoomorfa, recuperada de forma casual -mediante detector de metales- en una posible villa o establecimiento similar ubicado en las proximidades de la actual Fuenlabrada de los Montes (Badajoz). Es una zona donde, por el momento, la información arqueológica de que disponemos nos habla de un poblamiento más bien disperso -no existen ciudades conocidas en bastantes kilómetros a la redonda-, muy probablemente en relación con la cabaña ganadera, el control de rutas de comunicación -la más importante, el Guadiana- y, sin lugar a dudas, la riqueza ginecética, de la que sería buena prueba el motivo que adorna la pieza.

\section{Summary}

In this short article we report a Roman bronze chandelier with zoomorphic decoration discovered by chance -with a metal detector- in a possible villa or similar site situated near the present day village of Fuenlabrada de los Montes (Badajoz). It is an area where for the moment the archaeological information available suggests a scattered settlement -no cities or villages ara known for many miles around-probably related to a cattle breeding ranch, the communication routes control -the most important being the Guadiana- and undoubtedly the abundance of game of which the motif that decorates the piece would be a good indication.

El hallazgo tuvo lugar a comienzos de los años 90 en el lugar conocido como La Laguna, una zona adehesada, rica en agua, localizada unos dos kilómetros al Noroeste de Fuenlabrada de los Montes, dando vista al valle del río Guadiana, que discurre no

* Universidad de Córdoba.

* Universidad de León. 
demasiado lejano en dirección a Mérida. Es una pequeña loma entre montañas, que no se rotura desde hace al menos cincuenta años -lo que dificulta enormemente su prospección en superficie, si bien ha facilitado su desconocimiento hasta tiempos recientes-, y que hasta hace muy poco ha mantenido una considerable riqueza cinegética, hoy desplazada hacia los cotos cercanos, derivando su principal dedicación a la ganadería, principalmente vacuna.

La lucerna fue recuperada mediante detector de metales por un vecino de Fuenlabrada, quien la conserva en su colección particular y a quien agradecemos desde aquí las facilidades que nos ha prestado para su estudio. En la visita que realizamos al yacimiento en su compañía, al objeto de documentar con exactitud el lugar del hallazgo, no observamos restos de estructuras en superficie con excepción de algunas cimentaciones en diversos puntos donde un camino reciente ha eliminado el manto vegetal. Aunque con dificultad, parecen apreciarse varias alineaciones de cuarcitas irregulares -la roca de base en la zona inmediata es la pizarra, utilizada en todos los cercados vecinos, conformando con frecuencia un aparejo en "espina de pescado" realmente curioso-, identificables tal vez con los cimientos de alguna estructura, de características y cronología lógicamente indeterminadas.

Sí pudimos detectar, abarcando un espacio cercano a las dos hectáreas cierta proliferación de lateres, tegulae e imbrices, distribuidos en torno a una loma que adopta toda la apariencia de un tell. Sabemos que en las proximidades se han recuperado también mediante detector algunas monedas de cronología imprecisa, varios apliques de sítula o de muebles romanos, así como algunos recipientes de cocina en latón -en concreto, un cazo y un vaso de borde vuelto- que nos han llegado en muy mal estado de conservación, razón por la que hemos preferido aquí omitir su estudio.

Pretendemos, pues, con esta nota simplemente dar a conocer a la investigación una lucerna que, por su rareza, nos ha parecido lo bastante significativa como para ser objeto de un breve estudio monográfico, a insertar en ese puzzle aún en buena medida desconocido que representa el poblamiento romano en la región extremeña, de forma muy particular en el sector pacense nororiental.

Nos referimos a una zona que ya uno de nosotros contribuyó a dar a conocer hace unos años (VAQUERIZO, 1987), pero que en esencia continúa siendo prácticamente virgen para la investigación arqueológica, tanto de época prerromana como romana, pese a la publicación de algunos trabajos muy recientes que comienzan a dar idea de su potencialidad arqueológica, así como del carácter peculiar de sus gentes, inscritas en el marco de la provincia Baetica, aunque seguramente herederas de formaciones etnográficas y tal vez políticas de personalidad diferente a la propiamente andaluza como es el caso de la tan debatida Baeturia (GARCIA IGLESIAS, 1971; CORTIJO CEREZO, 87 ss.; AAVV, 1994).

En los últimos años se puede incluso hablar de un cierto boom bibliográfico en Extremadura, motivado en buena parte por la actividad desplegada desde la Univer- 
sidad y la Junta de Extremadura, el Museo Nacional de Arte Romano de Mérida y diversas iniciativas derivadas de algunas grandes construcciones públicas.

Sin embargo, y aunque estos estudios se refieren tanto a la etapa prerromana (AAVV, 1990; ORTIZ ROMERO, 1991; RODRIGUEZ DIAZ, 1989 y 1991; RODRIGUEZ, ORTIZ, 1989; RODRIGUEZ, ENRIQUEZ, 1992; BERROCAL, 1992; CELESTINO, JIMENEZ, 1993; AAVV, 1994), como a la de Romanización (BERROCAL, 1989-90; AAVV, 1991; GARCIA BELLIDO, 1993), la municipalización (AAVV, 1989; STYLOW, 1991), las vías (FERNANDEZ CORRALES, 1987), el territorio (FERNANDEZ CORRALES, 1988), o el poblamiento rural de época tardoantigua (CERRILLO, 1984), lo cierto es que la mayor parte de ellos se vienen centrando sobre la provincia de Cáceres y la mitad occidental de Badajoz, manteniendo un abandono casi total sobre la zona conocida tradicionalmente como "Siberia Extremeña", sólo paliado por algunos estudios recientes (PASTOR, PACHON, CARRASCO, 1992; AGUILAR, GUICHARD, 1993). Aún cuando, es ésta una zona que, no obstante y en nuestra opinión, habrá de deparar todavía importantes sorpresas, dada su riqueza ganadera y cinegética, así como su importante papel en las comunicaciones, en buena parte derivado de su cercanía al curso del Guadiana.

La pieza que ahora presentamos es una lucerna pseudo-plástica de bronce, con rostrum de volutas dobles o sin punta que termina en forma ojival, abriéndose en él un amplio orificio de iluminación, con $2^{\prime} 3 \mathrm{~cm}$. de diámetro. La margo, extensa y con desarrollo horizontal, se separa del discus -que ocupa en su totalidad una gran abertura de alimentación de $2 \mathrm{~cm}$. de diámetro - por una moldura. El ansa es anular, sin nịnguna molduración. La base, con un pie ligeramente resaltado, aparece plana, aunque algo rehundida hacia el centro.

Peso: 325 grs.

Altura máxima: $9 / 10 \mathrm{~cm}$., oscilación que se produce al no ser la pieza totalmente regular.

Altura máxima de la lucerna en sí misma: $4 / 5 \mathrm{~cm}$.

Diámetro máximo del discus: 5'5 cm.

Diámetro de la base: $3 \mathrm{~cm}$.

El asa sobresale $2 \mathrm{~cm}$. y ofrece también $2 \mathrm{~cm}$. de diámetro.

Presenta como decoración plástica la figura de un ciervo de amplia cornamenta recostado sobre la margo de la lucerna, en la zona de unión al ansa. Es de elaboración bastante sumaria, muy esquemático aunque expresivo a la vez, remarcando con especial incidencia precisamente la cuerna, de seis puntas muy desarrolladas y seis más incipientes, atributos que catalogan al animal casi como de primera categoría, cinegéticamente hablando. La cabeza se reduce a una simple prolongación de tendencia triangular, bastante alargada, de un esquematismo tal que recuerda enormemente a 
figuras similares de época prerromana (para una cumplida relación de los ejemplares de ciervo en todo tipo de manifestación artística documentados en la Península lbérica vid. CHAPA, 1985, 187 ss., donde puede encontrarse además una muy buena recopilación bibliográfica). Los cuemos y las orejas arrancan del cráneo con cierta lógica anatómica, pero los ojos se reducen a dos simples incisiones circulares y el hocico no existe, en un testuz de ángulos muy marcados y apariencia troncopiramidal. Denota la actuación de un artesano que seguramente trabaja de memoria, pero que reúne el encanto de lo popular y de la pureza de líneas.

La lucerna fue vaciada sobre un molde, retocada a cincel y finalmente bañada en bronce líquido, lo que ha originado una bella pátina bruñida sólo parcialmente conservada. El ciervo debió ser soldado, pero la insuficiente limpieza de este sector de la pieza nos impide asegurarlo con certeza. En cualquier caso, no observamos en toda la lámpara marca alguna de soldadura o apliques plásticos de ningún otro tipo.

Por el momento, no conocemos ningún paralelo exacto. Si hubiéramos de limitarnos a los parecidos formales, representaciones de este tipo se pueden rastrear en todo el Mediterráneo desde al menos el II milenio y de manera especial en el oriente del mismo, donde destacan los estandartes hititas, de una similitud asombrosa con la pieza que ahora nos ocupa (BLANCO, 1975, 281 ss., Figs. 137 y 139; BITTEL, 1976, 35 ss., Láms. 16,18 y 22). Y si es que no queremos realizar "arqueología-ficción", cayendo en anacronismos que no salva ni el más feroz difusionismo, podemos remontarnos a la propia etapa prerromana peninsular y de manera muy especial a tierras pacenses y portugueso-orientales, donde son muy abundantes desde el Período Orientalizante objetos metálicos diversos -casi siempre según parece relacionados con cuestiones rituales- en los que se representa el ciervo (GARCIA Y BELLIDO, 1957 y 1958; CHAPA, 1985, 187 ss.).

Sin embargo, para la época romana -y salvo que planteemos la posibilidad de adscribir a esta cronología algunas de las piezas a que acabamos de aludir, teóricamente prerromanas aunque siempre recuperadas fuera de todo contexto arqueológico- el ejemplar de mayor semejanza que conocemos es una lámpara en cerámica, procedente de Cartago, identificable con la forma Deneauve $X B$ y datada en el siglo III de la Era, si bien lo que se representa en ella no es un ciervo, sino un león (DENEAUVE, 1969, $\mathrm{N}^{\circ}$ 1066). Mucho más lejanamente, sobre todo en la forma, la recuerda otra pieza del Museo de Bellas Artes de Budapest con un perro, a la que no se asigna cronología (SZENTLELEKY, 1969, N²83).

De esta manera, los mayores problemas que se nos plantean para definir la cronología de la lucerna de Fuenlabrada derivan lógicamente de la ausencia total de contexto arqueológico que acompañara al hallazgo, así como de una escasez de paralelos que no nos permiten una fácil filiación por asociación y/o comparación. El rasgo formal que en una primera impresión parece más definitorio, la presencia de volutas dobles, deja de serlo desde el momento en que éstas se dan en ejemplares de bronce remon- 
tables tanto al período que abarca desde la época helenística al final del siglo I d.C., como al centrado en los siglos que definen la Antigüedad Tardía (siglos IV-VI d.C.).

Con todo, tomando en consideración las características que algunos estudiosos atribuyen a uno y otro período (CONTICELLO, CAROLIS, 1986, 42-43), parece más probable su inclusión en el segundo de esos momentos -hipótesis en la que parecen abundar los objetos metálicos de menaje recuperados en relación con la lucerna-, coincidiendo con un presunto desarrollo del poblamiento rural en la zona relacionado con los valores medioambientales de la misma y similar al señalado para otras zonas de Extremadura (CERRILLO, 1984; FERNANDEZ CORRALES, 1988; AGUILAR, GUICHARD, 1993).

En este sentido, y pese a la cronología tardía que creemos corresponde a la pieza, no queremos caer en explicaciones rebuscadas en la interpretación de su decoración. La relativa abundancia de representaciones prerromanas de cérvidos en la zona ha sido atribuida tradicionalmente al carácter sagrado de este animal para los lusitanos, derivado de su posible asimilación con una "deidad indígena lusitana, que estaría relacionada no sólo con la caza, sino con su expresión suprema, la guerra de la que la caza es sólo un preludio". Divinidad indígena -tal vez Ataecina- identificable muy probablemente con Artemis-Diana (GARCIA Y BELLIDO, 1957, 129 ss.).

A pesar de ello, preferimos desechar por el momento un contenido religioso en sentido estricto para la pieza -ni siquiera de la posible comparación/asimilación del ciervo con el fiel en el sentido cristiano- para definirnos por una lectura mucho más inmediata: la selección, por compra o encargo, de este tipo de objetos por personas que los consumían y disfrutaban de acuerdo con sus gustos y su propio sentido de la estética (AMARE, 1987, 45-46).

De acuerdo con esta idea, y dadas las propias características de la zona, no creemos descabellado reconocer en la persona que la disfrutó a un aficionado de la venatio. Respondería así a una moda que, aplicada a la caza de esta misma especie animal, nos ha llegado reflejada para la misma época en otras muchas manifestaciones artísticas como la pintura mural (ABAD CASAL, 1982, 347 ss., Fig. 115) o la decoración musivaria, donde este tipo de temas representan además un símbolo importantísimo de status (BLAZQUEZ, 1993, 236). Todo lo cual nos lleva además a considerar la lucerna como de fabricación autóctona, quizá no in situ, pero sí en la zona. 


\section{Bibliografía}

AAVV (1989), Aspectos de la colonización y municipalización de Hispania, Cuadernos Emeritenses 1, Mérida.

AAVV (1990), La cultura tartésica y Extremadura, Cuadernos Emeritenses 2, Mérida.

AAVV (1991), Extremadura Arqueológica II. Actas de las I Jornadas de Prehistoria y Arqueología en Extremadura (1986-1990), Mérida-Cáceres.

AAVV (1994), Celtas y túrdulos: La Beturia. Actas del Coloquio celebrado en el Museo Nacional de Arte Romano de Mérida los días 25 y 26 de mayo de 1994. En prensa.

ABAD CASAL, L. (1982), Pintura romana en España, 2 vols., Univ. de Alicante-Univ. de Sevilla.

AGUILAR, A.; GUICHARD, P. (1993), Villas romaines d'Estrémadure. Doña María, La Sevillana et leur environnement, Casa de Velázquez, Madrid.

AMARE, M. T. (1987), Lucernas romanas: generalidades y bibliografía, Zaragoza.

BERROCAL RANGEL, L. (1989-1990), "Cambio cultural y romanización en el suroeste peninsular", Anas 2-3, Museo Nacional de Arte Romano, Mérida, pp. 103-122.

- (1992), Los pueblos célticos del Suroeste de la Península Ibérica, Complutum 2-Extra, Ed. Complutense, Madrid.

BITTEL, K. (1976), Los Hititas, Colección Universo de las Formas, Ed. Aguilar, Bilbao.

BLANCO FREIJEIRO, A. (1975), Arte Antiguo del Asia Anterior, Sevilla.

BLAZQUEZ, J. M. (1993), Mosaicos romanos de España, Ed. Cátedra, Madrid.

CELESTINO, S.; JIMENEZ, F. J. (1993), El palacio-santuario de Cancho Roano. 1 V. El Sector Norte, Badajoz.

CERRILLO MARTIN DE CACERES, E. (1984), La vida rural romana en Extremadura, Publ. de la Univ. de Extremadura, Cáceres.

CHAPA BRUNET, T. (1985), La escultura ibérica zoomorfa, Min. de Cultura, Madrid.

CONTICELLO, M.; CAROLIS, E. de (1986), Le lucerne di bronzo, Ciudad del Vaticano.

CORTIJO CEREZO, M. L. (1993), La administración territorial de la Bética romana, Córdoba.

DENEAUVE, J. (1969), Lampes de Carthage, Paris.

FERNANDEZ CORRALES, J. M. (1987), El trazado de las vías romanas en Extremadura, Madrid.

- (1988), El asentamiento romano en Extremadura y su análisis espacial, Câceres.

GARCIA BELLIDO, A. (1957), "El jarro ritual lusitano de la Colección Calzadilla", AEspA XXX, Madrid, pp. 121-138.

— (1958), "De nuevo sobre el jarro ritual lusitano, publicado en AEArq 30, 1957, 121 ss.", AEspA 31, Madrid, pp. 153-164.

GARCIA BELLIDO, M. P. (1993), "Sobre las dos supuestas ciudades de la Bética llamadas Arsa. Testimonios púnicos de la Beturia túrdula", Anas IV-V (1991-1992), Mérida, pp. 81-92.

GARCIA IGLESIAS, L. (1971), "La Beturia, un problema geográfico de la Hispania Antigua", AEspA XLIV, Madrid, pp. 86-108.

SZENTLELEKY, Th. (1969), Ancient Lamps, Amsterdam.

ORTIZ ROMERO, P. (1991), "Excavaciones y sondeos en los recintos tipo torre de La Serena", Extremadura Arqueológica II, Mérida-Cáceres, pp. 301-318.

PASTOR, M.; PACHON, J. A.; CARRASCO, J. (1992), Mirobriga. Excavaciones Arqueológicas en el "Cerro del Cabezo" (Capilla, Badajoz). Campañas 1987-1988, Mérida. 
RODRIGUEZ DIAZ, A. (1989), "La Segunda Edad del Hierro en la Baja Extremadura: problemática y perspectiva en torno al poblamiento", Saguntum. P.L.A.V. 22, Univ. de Valencia, pp. 165-224.

—- (Coord.) (1991), La Ermita de Belén (Zafra, Badajoz). Campaña 1987, Mérida.

RODRIGUEZ, A.; ORTIZ, P. (1989), "Poblamiento prerromano y recintos ciclópeos de La Serena (Badajoz)", CUPAUAM 17, Madrid, pp. 45-65.

RODRIGUEZ, A.; ENRIQUEZ, J. J. (1992), "Necrópolis protohistóricas en Extremadura”, Congreso de Arqueología Ibérica. Las Necrópolis, Madrid, pp. 1-26.

STYLOW, A. U. (1991), "El municipium Flavium V (...) de Azuaga (Badajoz) y la municipalización de la Baeturia turdulorum", Studia Storica. Historia Antigua IX, Salamanca, pp. 11-27.

VAQUERIZO GIL, D. (1987), "Primeros resultados de la investigación arqueológica en la llamada Siberia Extremeña (Badajoz)", XVIII CNA, Zaragoza, pp. 813-832. 

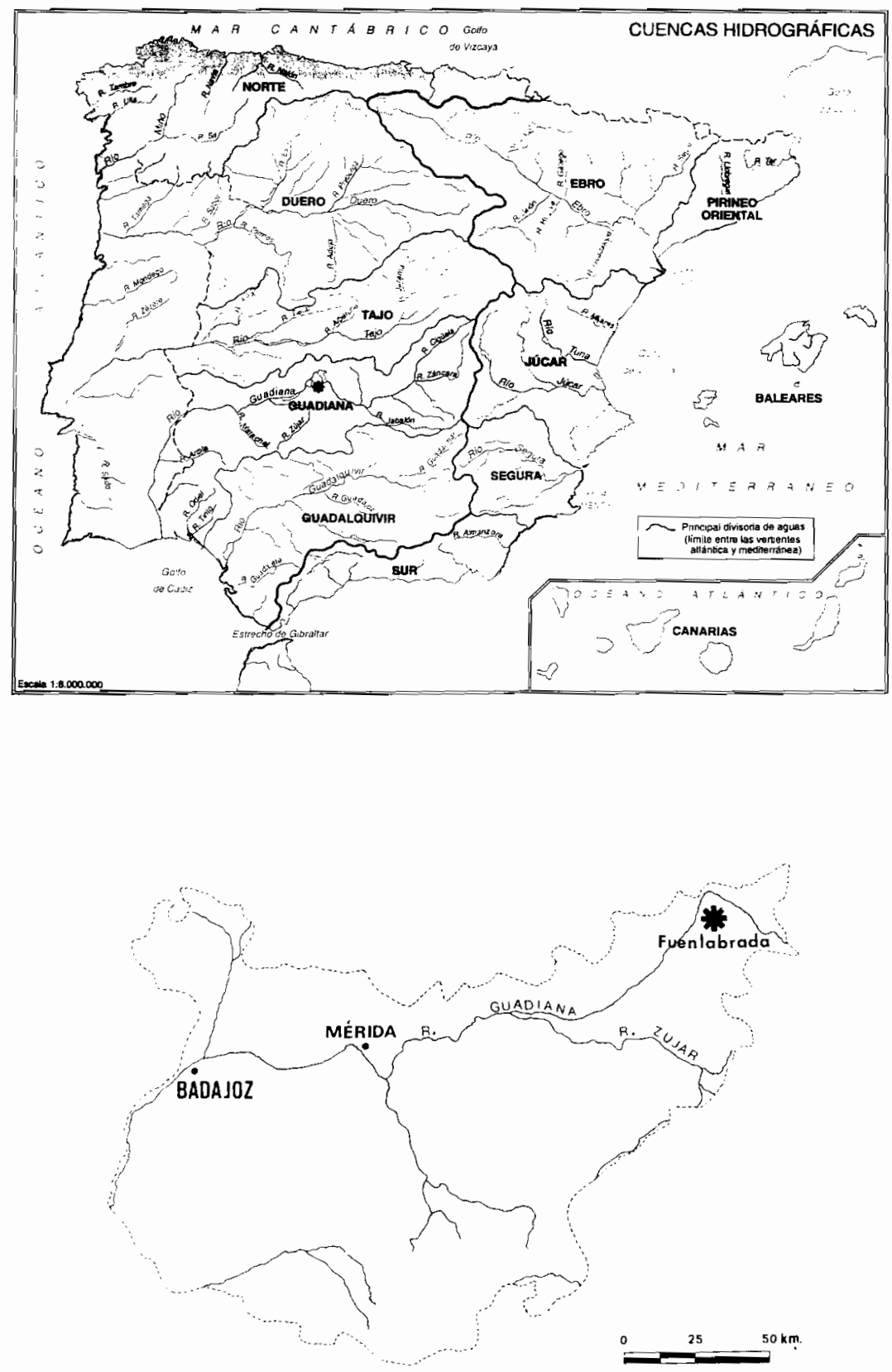

Fig. 1: A) Localización de Fuenlabrada de los Montes en relación con la cuenca del rio Guadiana (según AAVV, 1993, 18). B) La misma localidad en el marco de la actual provincia pacense (plano tomado de PASTOR, PACHON, CARRASCO, 1993, 175, Fig. 1-A). 

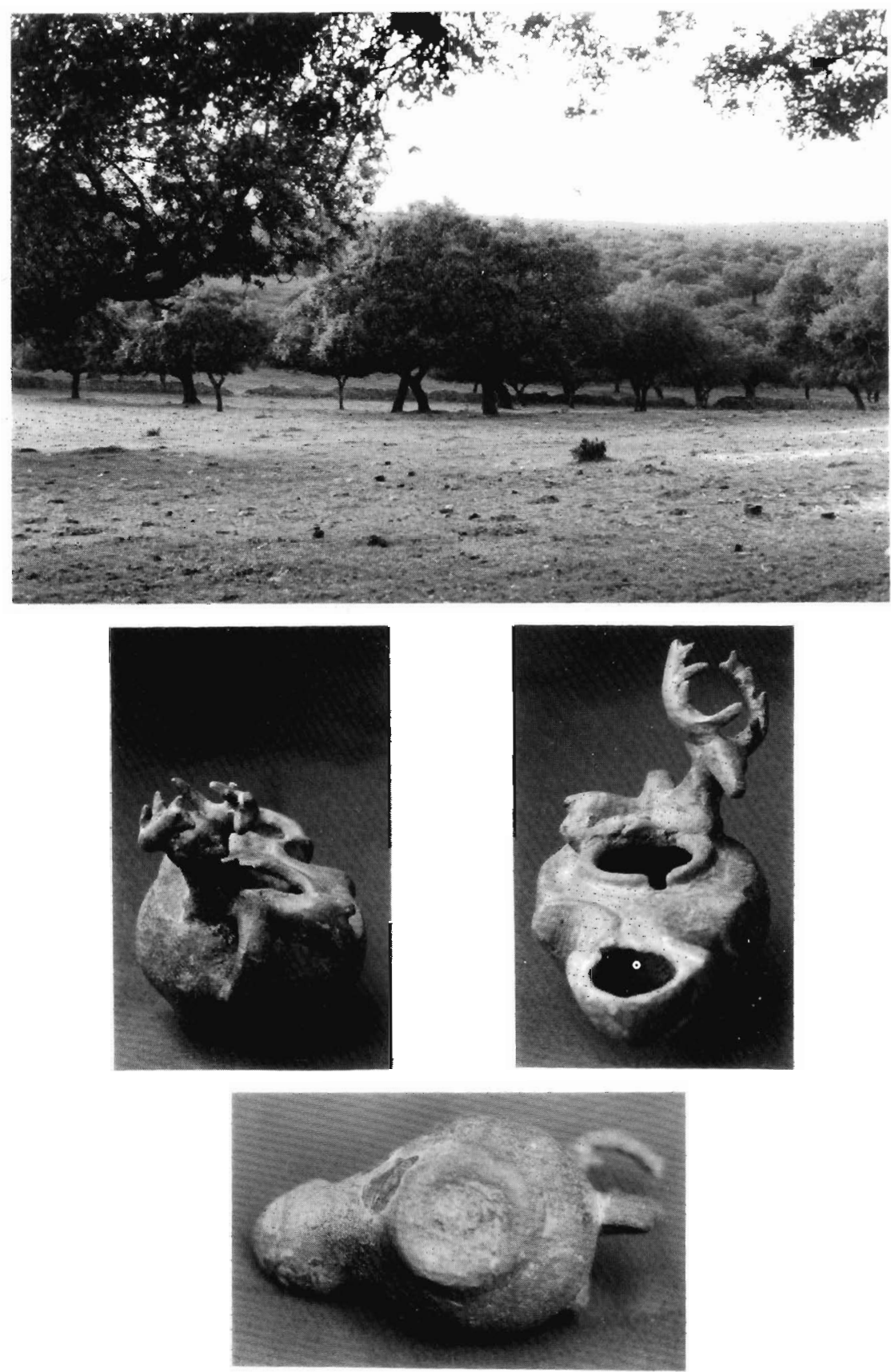

Lám.1: A) Aspecto general del yacimiento, en un paisaje típicamente adehesado muy próximo al curso del Guadiana (Fotog. D. VAQUERIZO). B) Lucerna de La Laguna, Fuenlabrada de los Montes (Fotog. D. VAQUERIZO). 

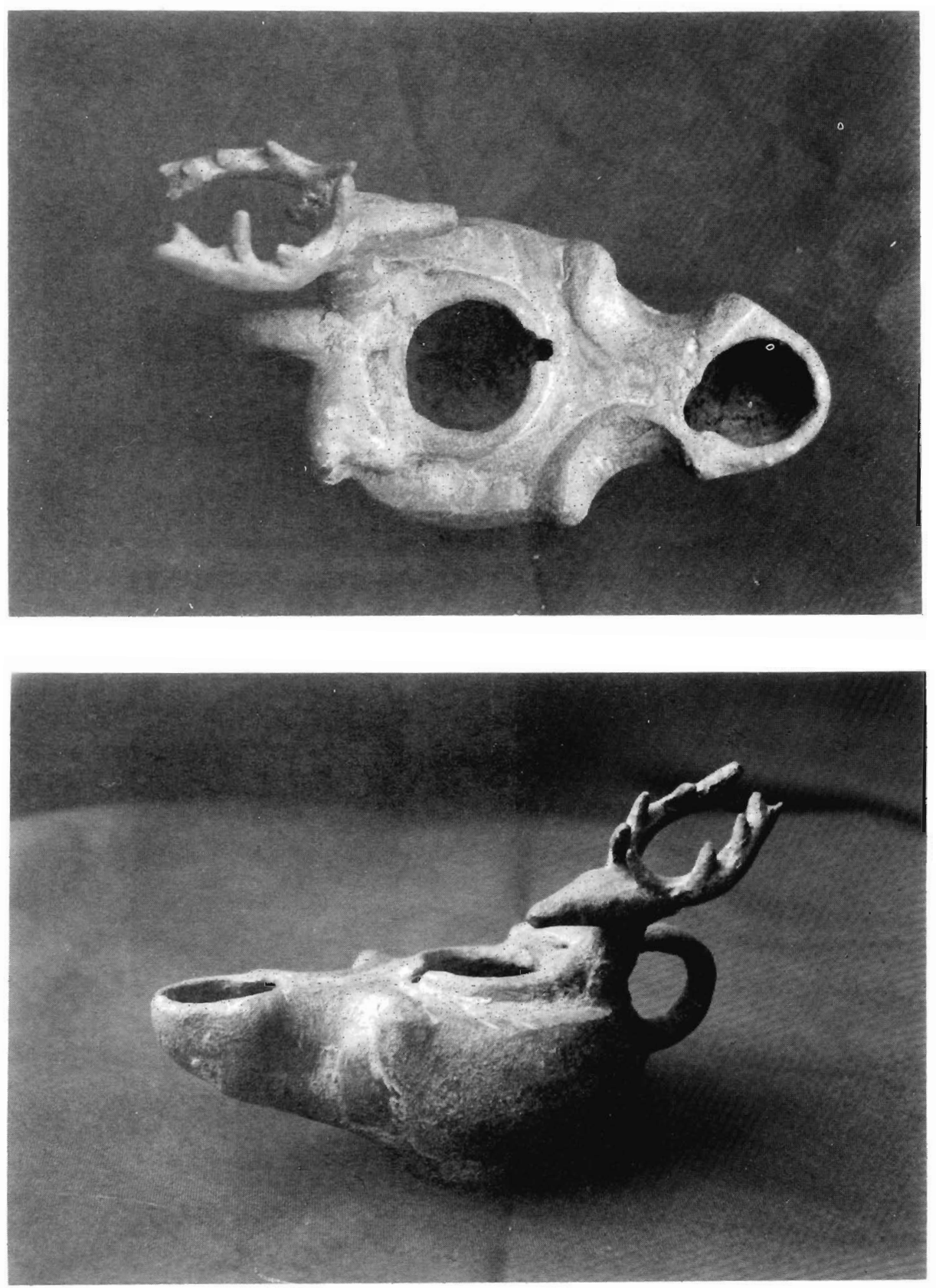

Láms. 2, 3 y 4: Lucerna de La Laguna, Fuenlabrada de los Montes (Fotog. D. VAQUERIZO). 


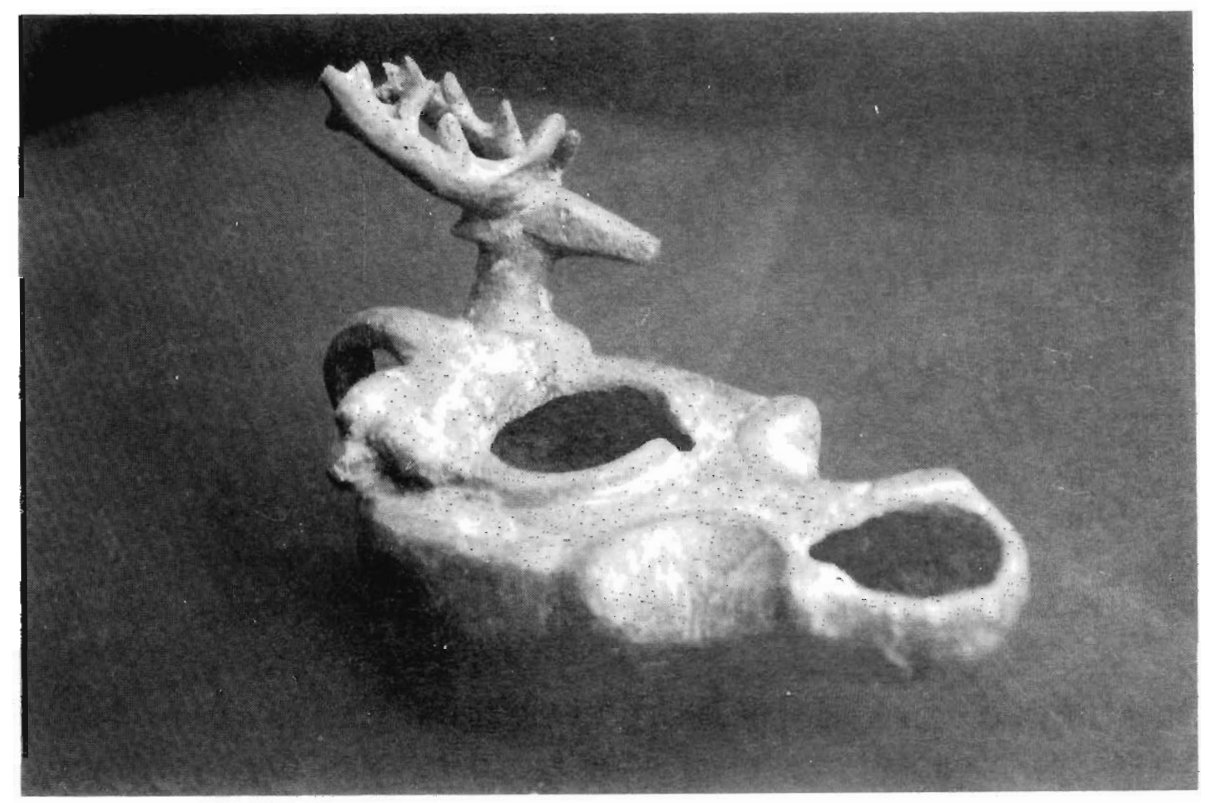

Lám. 4.

--- Grupo de investigación P.A.I. HUM 236 | http://www.arqueocordoba.com/publ/anales.htm --- 